\title{
Problems Associated With the Need for Standardization in Clinical Spectrophotometric and Fluorometric Measurements
}

\author{
James R. Penton, Graham M. Widdowson, and George Z. Williams \\ Research Institute of Laboratory Medicine Institute of Medical Sciences, San Francisco, Calif. 94115
}

(June 5, 1972)

\begin{abstract}
There is a growing demand in clinical chemistry for analyses to be performed in a manner allowing comparisons of results among laboratories and, from time to time, in the same laboratory. Reliable comparability requires adequate procedures of standardization for spectrophotometric and fluorometric instruments and methods. Problems with chemical and instrumental standardization are discussed.

For assays where the substance to be measured is available in suitable form, primary chemical standardization is justifiably popular. Relatively unsophisticated instrumentation can be used to compare measurements of unknown samples with such standards. Because primary standards meeting all necessary criteria are not available for many assays of clinical significance, standardization must depend on precision and accuracy of the instrumentation used, and on accurately compiled values of chemical-optical properties for the materials of interest. The task of compilation is outside the capability of the routine laboratory and should be provided by a reliable central agency. If an individual laboratory is to use the agency's compiled values, that laboratory must have available precise, accurate and reasonably inexpensive instrumentation along with reliable absorbance, fluorescence, and wavelength calibration standards.
\end{abstract}

Key words: Clinical standards; standard reference materials; standardization, spectrofluorometric; standardization, spectrophotometric.

\section{Introduction}

Spectrophotometric analyses in the routine clinical chemistry laboratory are performed within a unique framework. This framework imposes conditions responsible for many of the problems of inaccuracy and lack of standardization in clinical chemistry. The factors comprising this framework include:

a. Workload-many clinical chemistry laboratories are performing in excess of a quarter of a million analyses each year, and this number has been growing in the United States at a rate as high as 15 percent per annum.

b. Time-to enable prompt action by a physician, results are nearly always required on the day the specimen is collected; quite often results are needed within an hour. A twenty-four hour service must usually be maintained.

c. Range of analyses - as many as 40 different determinations are commonly available on request with a wide range of "special investigations" available by arrangement. d. Type of sample-analyses are most commonly performed on serum or plasma, a viscous, proteinrich complex matrix available in restricted quantity.

e. Cost-clinical chemistry determinations contribute to the rising costs of health care; therefore the laboratory director must be cost-conscious, though not at the expense of precision and accuracy.

f. Personnel-the competent clinical chemistry technician must master a growing number of techniques, and operate a wide range of instrumentation that depends on many different principles.

As long ago as 1947, Belk and Sunderman [1] ${ }^{1}$ suggested the need for standardization in the clinical chemistry laboratory. They distributed aqueous solutions of glucose, chloride, urea and calcium, serum for measurements of total protein and albumin, and citrated whole blood for measurements of hemoglobin concentration, to a number of laboratories in Pennsylvania. They revealed wide discrepancies between results from different laboratories analyzing identical

${ }^{1}$ Figures in brackets indicate the literature references at the end of this paper. 
specimens. For example, 27 of 41 laboratories obtained urea nitrogen results more than $5 \mathrm{mg} / 100 \mathrm{ml}$ from the actual concentration of $45 \mathrm{mg} / 100 \mathrm{ml}$. That poor accuracy of results was a worldwide problem was demonstrated by similar reports from Australia [2], Britain [3], Canada [4], and New Zealand [5].

A major change in the operation of most routine clinical chemistry laboratories during the last 15 years has resulted from the introduction of mechanized and automated analytical equipment, both of the discrete sample and continuous flow type. That the introduction of such equipment can improve day-today precision of laboratory results has been demonstrated by Mitchell [6]. Gowenlock [7], reporting on an interlaboratory trial in Britain in 1965, concluded that apart from potassium determinations in serum, laboratories using AutoAnalyzer ${ }^{2}$ (Technicon Instruments Corporation, Tarrytown, N.Y.) methods produced more consistent results. Many laboratories now have quality control schemes usually checking the precision of results either by using pooled human sera, commercial control sera or, in the United States, by participating in the College of American Pathologists' Quality Assurance Service.

It might be assumed that the introduction of mechanized and automared equipment, together with the use of quality control techniques, would by now have improved accuracy and thus interlaboratory agreement. The results of the latest surveys are not always reported in a comparable way to those of Belk and Sunderman [1], making such judgment difficult. For example, the College of American Pathologists' Quality Evaluation Program for 1971 divided the results for a given determination into groups according to the type of analytical method used; no overall range, or range for results within a group, was given. The results of the national quality control scheme in Britain, however, give the individual results obtained by each laboratory. Figure 1 (taken from the British scheme for a week in September 1969) shows the wide distribution of results from different laboratories when aliquots of identical serum specimens were analyzed for uric acid.

Even if there has been a slight improvement in the accuracy of results since 1947, the performance of clinical chemistry laboratories still falls far below that which should be expected.

A precise analytical method is not necessarily accurate and herein probably lies a major cause of the problem. Lack of accuracy in spectrophotometric methods of analysis may be caused by several factors including lack of suitable standards, inadequate colorimeters and spectrophotometers; and poor specificity of the chemical reaction. The latter factor is outside the scope of this discussion. Though there is frequently an interdependence between the chemical and instrumental aspects of analysis, we will consider them separately.

\footnotetext{
${ }^{2}$ In order to adequately describe materials and experimental procedures, it was occasionally necessary to identify commercial products by manufacturer's name or label. In no instances does such identification imply endorsement by the National Bureau of Standards, nor does it imply that the particular product or equipment is necessarily the best available for that purpose.
}

\begin{tabular}{|c|c|c|c|c|c|}
\hline \multicolumn{3}{|c|}{ URIC ACID mg/100 mI } & \multicolumn{3}{|c|}{ NUMBER OF LABORATORIES } \\
\hline Less than minimum & $\uparrow$ & & 11 & I & \\
\hline 3.1 & TO & 3.19 & 3 & I & $x x x$ \\
\hline 3.2 & TO & 3.29 & 3 & I & $x x x$ \\
\hline 3.3 & TO & 3.39 & 0 & I & \\
\hline 3.4 & TO & 3.49 & 0 & I & \\
\hline 3.5 & TO & 3.59 & 7 & I & $X X X X X X X$ \\
\hline 3.6 & TO & 3.69 & 6 & I & $X X X X X X$ \\
\hline 3.7 & TO & 3.79 & 3 & I & $x x x$ \\
\hline 3.8 & TO & 3.89 & 10 & I & $X X X X X X X X X X$ \\
\hline 3.9 & TO & 3.99 & 7 & I & $x X X X X X X$ \\
\hline 4.0 & TO & 4.09 & 11 & I & $X X X X X X X X X X X X$ \\
\hline 4.1 & TO & 4.19 & 18 & I & $X X X X X X X X X X X X X X X X X X X X$ \\
\hline 4.2 & TO & 4.29 & 17 & I & $X X X X X X X X X X X X X X X X X X X X$ \\
\hline 4.3 & TO & 4.39 & 13 & I & $X X X X X X X X X X X X X X$ \\
\hline 4.4 & TO & 4.49 & 14 & I & $X X X X X X X X X X X X X X X X$ \\
\hline 4.5 & TO & 4.59 & 12 & I & $X X X X X X X X X X X X X$ \\
\hline 4.6 & TO & 4.69 & 6 & I & $X X X X X X$ \\
\hline 4.7 & TO & 4.79 & 3 & I & $x x x$ \\
\hline 4.8 & TO & 4.89 & 8 & I & $X X X X X X X X$ \\
\hline 4.9 & TO & 4.99 & 3 & I & $x x x$ \\
\hline 5.0 & TO & 5.09 & 2 & I & $x x$ \\
\hline Greater than maximu & 1 & & 7 & I & $\ldots$ \\
\hline
\end{tabular}

FIGURE 1. Histogram of uric acid results obtained when aliquots of a serum specimen were analyzed by 164 laboratories in the United Kingdom, during September, 1969. Courtesy of the Department of Health and Social Security, United Kingdom Committee on Standards and Quality Control in Clinical Biochemistry.

\section{Instrumental Considerations}

To function appropriately within the described framework of clinical chemistry requirements, a spectrophotometric analytical system must satisfy certain criteria. Those criteria concerned with economics, i.e., initial cost, operating cost and analytical rate, will, for the purpose of this discussion, be considered satisfied; only those criteria directly affecting standardization will be considered. Generally, these criteria are:

a. Instrument specifications adequate for standardization of the analysis.

b. Instrument meets specifications.

c. Instrument can be easily operated to verified attainable performance.

d. Instrument performance routinely verified in quality control program.

e. Operators understand behavior of instrument if measurement limits are exceeded.

Satisfaction of the criterion of adequate specification implies a thorough knowledge of the measurement characteristics of the instrumental system and of the interaction of those characteristics with those of the chemical system. It is essential to know: the range of absorbance which the spectrophotometric device will be required to measure, the wavelength at which measurements must be made, spectral band widths of absorption spectra to be investigated, temperature 
characteristics of both instrumental and chemical systems, and calibration stability of the instrumental system.

Once the necessary characteristics of the instrument system have been defined, performance must be verified. An adequate evaluation of instrument performance requires reference materials of independently verified quality. These materials may be used to check wavelength accuracy, spectrophotometric linearity and, if necessary, spectrophotometric accuracy.

\section{A. Wavelength}

Several wavelength tests are available. In instruments where its use is convenient, Rand [8] recommended the mercury vapor lamp as a calibration source because of its many well defined emission lines. Where use of a mercury vapor lamp is inconvenient because of mechanical considerations, the sharp absorption bands of didymium and holmium oxide glass filters $[8,9]$ can furnish useful calibration points. These same filters, however, must not be used for spectrophotometric linearity and accuracy tests. Their narrow absorption band widths, which suit them for wavelength testing, make their transmission much too sensitive to wavelength setting accuracy.

\section{B. Spectrophotometric Linearity}

Reule [10] pointed out that spectrophotometric linearity is a necessary, but not a sufficient, condition for spectrophotometric accuracy. He noted that scale readings could be linear with light absorbance change but differ from true absorbance by a nonunity factor. On this basis, he objected to the use of absorbing materials to test spectrophotometer performance; and he suggested a precise test method using carefully controlled additions of light. Reule's method is not suitable for general clinical laboratory use because of its complexity. However, the information he presents is fundamental to understanding of linearity and accuracy tests.

Only spectrophotometric linearity is required in many clinical chemistry assays. In these cases, Reule's objection to tests based on substances which obey the Lambert-Beer law is negated and stable solutions of substances with broad absorption bands near the wavelengths of interest may be employed. Acidic solutions of cobalt ammonium sulfate and alkaline solutions of potassium chromate [11] have been suggested by NBS. Rand [8] favored acidic potassium dichromate (available from NBS as Standard Reference Material 136c). Buffered solutions of reduced nicotinamide adenine dinucleotide (NADH) may be useful if their marginal stability is taken into account. Solutions carefully prepared from materials of known ancestry can serve as useful spectrophotometric linearity benchmarks in both wide and narrow bandwidth spectrophotometers.

An instrumental characteristic which can markedly reduce the linear range of a photometric device is stray light [12]. Results of an experiment in our laboratory illustrate the effect of stray light and are shown in figure 2. These data were obtained on an AMINCO Rotochem (American Instrument Company, Silver Spring, Md.) from determinations of the transmittance of solutions of NADH in $0.1 \mathrm{~mol} \cdot 1^{-1}$ phosphate buffer $(\mathrm{pH}=7.4)$. Measurements were taken at $340 \mathrm{~nm}$, both with and without a minus-visible $360 \mathrm{~nm}$ cutoff filter in the photo detector window. The fall-off of linearity seen at low transmittance without the filter results from stray heterochromatic light "sneaking past" the monochromating element and into the photo detector without being attenuated by the absorption bands of the sample. The filter recommended by the manufacturer in this example is typical of the "stray light" and "order-sorter" filters found on prism and grating monochromators. Mis- or disuse of such filters can degrade the performance of even the best spectrophotometer to a level far below the specifications upon which its procurement was based.

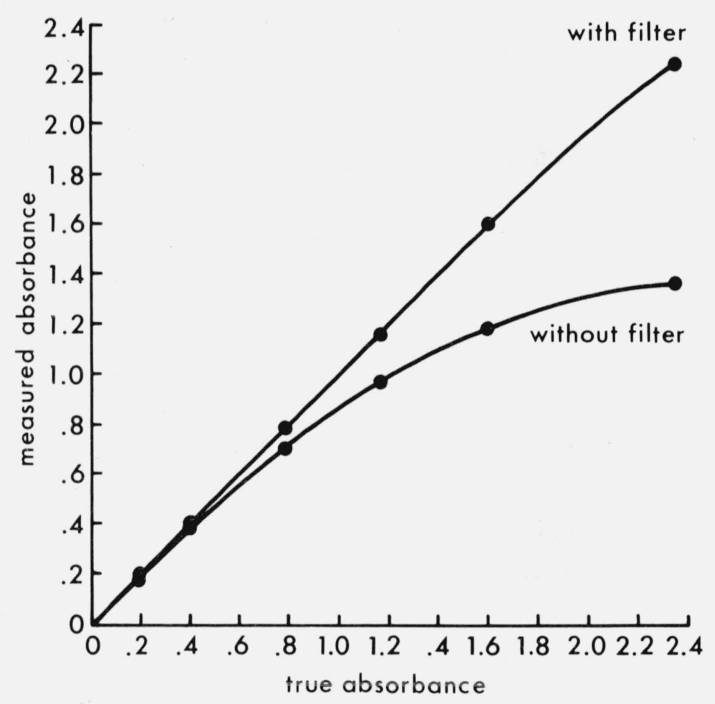

Figure 2. Absorbance of NADH solutions measured at $340 \mathrm{~nm}$ with and without stray light filter.

\section{Spectrophotometric Accuracy}

When the goal of instrument testing is verification of spectrophotometric accuracy, several pitfalls appear beyond those associated with linearity. Even though linearity tests may be satisfied in characterizing the spectrophotometric performance of wide bandwidth devices, in most cases these instruments are not capable of spectrophotometric accuracy. The data in figure 3 were obtained in our laboratory from solutions of buffered $p$-nitrophenol measured at $404 \mathrm{~nm}$ in an AMINCO Rotochem. The curves show that increasing spectral bandwidth causes apparent absorbance decreases from the "true" absorbance measured on the Beckman DU (Beckman Instruments Inc., Fullerton, Calif.) at $0.6 \mathrm{~nm}$ bandwidth; linearity remains good. If the instrument under test is of adequately narrow 


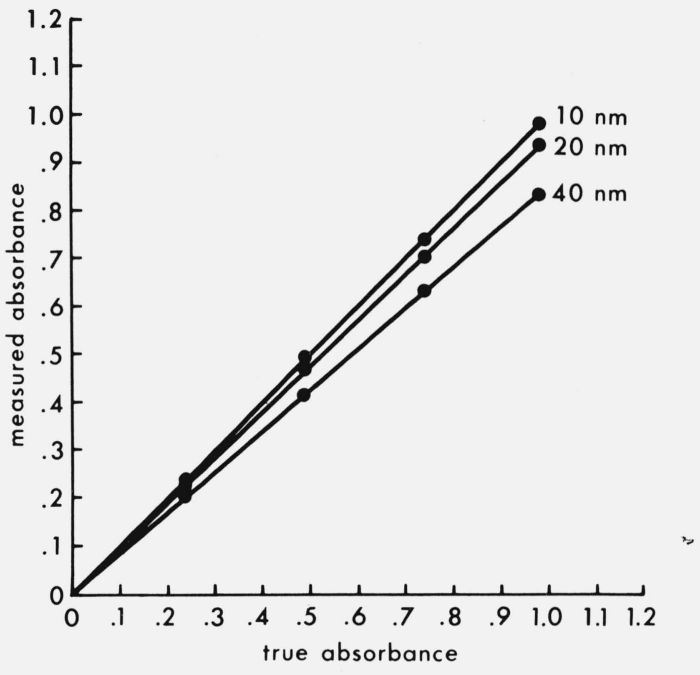

Figure 3. Absorbance of $p$-nitrophenol solutions measured at $404 \mathrm{~nm}$ with bandwidth of $40 \mathrm{~nm}, 20 \mathrm{~nm}$ and $10 \mathrm{~nm}$; plotted against true absorbance.

bandwidth to warrant investigations involving spectrophotometric accuracy, then the materials used to assess this must be considered. In obtaining the $\mathrm{NADH}$ results described in figure 2 , we noticed that the measured absorbances were lower than predicted from weight concentration and the accepted molar absorptivity of NADH reported by Horecker and Kornberg [13]. Solutions of NADH (Sigma > $>98 \%$ purity) from two lot numbers were prepared in phosphate buffer, and their absorbances measured at $340 \mathrm{~nm}$ on a Beckman DU spectrophotometer. Our calculated molar absorptivities were approximately two-thirds of the expected value. When the indirect determination based on enzymatic conversion of nicotinamide adenine dinucleotide (NAD) to NADH described in [13] was duplicated, molar absorptivities in good agreement with the reference value were obtained. The source of this discrepancy has not yet been resolved, but it is obvious that direct use of $\mathrm{NADH}$ as a spectrophotometric accuracy-test material must be approached with caution.

Slavin [14] discussed the relative merits of liquid and solid test materials for spectrophotometric accuracy assessment and concluded that the requirements of convenience and stability favored use of solid materials. He described applications of the Chance-Pilkington (England) ON-10 neutral filter glass and of perforated metal screen filters. Copeland, King and Willis [15] described the use of the NBS carbon yellow filter glass as absorbance standard for the $540 \mathrm{~nm}$ assay of cyanmethemoglobin but lamented the strong wavelength dependence $(0.0083$ $\mathrm{A} / \mathrm{nm}$ ) of the filter at the desired wavelength.

Wavelength dependence is a criticism common to most reference materials proposed for use in spectrophotometric accuracy tests, especially when such tests are conducted in the environment of the clinical chemistry laboratory. Each material requires con- siderable discretion in choice of wavelength range for which it is used. If the material is used where its absorbance is strongly wavelength-dependent, then seemingly trivial errors in wavelength calibration can lead to decidedly nontrivial errors in spectrophotometric accuracy assessments. Since the Beckman DU used by Copeland, King, and Willis [15] has a wavelength accuracy of about $\pm 0.4 \mathrm{~nm}$, this measurement on the NBS carbon yellow filter could have been in error by $0.0035 \mathrm{~A}$ units from wavelength inaccuracy alone.

\section{Functional Considerations}

Problems resulting from inadequate wavelength accuracy, spectrophotometric linearity and spectrophotometric accuracy may be minimized by using extreme care in specifying and verifying a spectrophotometric system, but we ask what are the implications of poor performance of these functions in the nonideal world of the clinical chemistry laboratory.

In enzyme assay by kinetic measurements, the rate of substrate conversion is calculated from molar absorptivity and absorbance change with time. When substrates and conversion products have relatively narrow spectral bandwidth absorption curves, accurate calculation of the concentration from absorbance and molar absorptivity will depend on accuracy of wavelength setting. To illustrate the effect of wavelength dependence, we performed assays of serum alkaline phosphatase using conversion of $p$-nitrophenyl phosphate to $p$-nitrophenol. Measurements of kinetic rates were made at the correct wavelength of $404 \mathrm{~nm}$ and at "erroneous" wavelengths of $414 \mathrm{~nm}$ and $424 \mathrm{~nm}$. The results in table 1 show that the apparent activities are considerably lowered by errors in wavelength.

TABLE 1. Effects of wavelength on apparent activity of alkaline phosphatase

\begin{tabular}{cccc} 
& \multicolumn{3}{c}{ Activity* $\left(\right.$ I.U./L) at $30{ }^{\circ} \mathrm{C}$} \\
\cline { 2 - 4 } Sample number & $404 \mathrm{~nm}$ & $414 \mathrm{~nm}$ & $424 \mathrm{~nm}$ \\
1 & 38.3 & 35.4 & 28.8 \\
2 & 68.9 & 64.3 & 51.4 \\
3 & 59.8 & 56.3 & 45.4 \\
4 & 38.3 & 34.7 & 28.5 \\
5 & 57.4 & 53.2 & 41.5 \\
6 & 50.0 & 46.3 & 36.6
\end{tabular}

*Activities are the average of two assays.

Even in the assays in which comparison measure ments are made between standard and unknown samples, spectrophotometric linearity is required, except in the trivial case where the standard and the unknown have exactly the same light transmittance. With instruments of nominally acceptable linearity, there is still danger that unknown samples may be encountered with transmittance outside the verified range of linearity, and routine treatment of such samples will cause reduction in precision of the measurement. This loss of precision stems from either 
or both of two sources. The more severe effect results from measuring in a range of transmittance below that for which the spectrophotometer is linear. Necessity for verification of linearity for each individual instrument is obvious because some commonly used clinical colorimeters have significant deviations from linearity at absorbances as low as 0.5 (Association of Clinical Biochemists' (A.C.B.) reports [16, 17]).

Another serious, but less apparent, source of precision loss results from measuring at transmittances far removed from 0.368 (Absorbance $=0.434$ ). Near this transmittance Beer's law calculations yield the lowest relative concentration error $(\Delta C / C)$ for any given uncertainty in transmission measurement [18, 19]. Willard, Merritt and Dean [20] show the relative concentration error, resulting from a transmittance measurement uncertainty of 0.4 percent, to range from 8.2 percent at $0.95 T$ through 1.1 percent at $0.40 T$ to 8.7 percent at $0.01 T$.

The assay of enzymes by kinetic rate determination often requires measurement of small changes in absorbance. Many spectrophotometers commonly used for direct concentration assay do not have absorbance resolution adequate to measure these small differences precisely. Absorbance is proportional to concentration of the absorbing species in direct measurements. Therefore, for a given relative error in absorbance measurement, the relative error in concentration is the same; e.g., if an absorbance measurement is $0.500 \pm 0.002$, then the relative error is $\pm(100$ percent $\times 0.002 / 0.500)$ or \pm 0.4 percent, and the relative error in concentration calculated from this absorbance measurement also will be \pm 0.4 percent.

However, if the same spectrophotometric error $( \pm 0.002)$ is present in an instrument used to measure a kinetic rate, the relative error on the assay results may be much worse. When the enzyme aspartate aminotransferase (SGOT) is determined by a common kinetic rate method [21], the absorbance change observed for samples from normal subjects is of the order of 0.01 per min at $30{ }^{\circ} \mathrm{C}$. Typical absorbances for two readings taken 2 min apart are 1.000 and 0.980 and the difference, 0.02 . In the worst case, when the measurement errors for each of the two readings are additive, the relative error could be $\pm(100$ percent $\times 0.004$ / $0.020)= \pm 20$ percent. The fundamental problem is that of measuring precisely small differences in large numbers. Thus, small relative errors in each of the two measurements can lead to large relative errors in the value derived from their difference.

This fundamental problem of precisely measuring small differences is further aggravated by the widespread use of instruments with digital display or printout of readings. While digital displays do much to reduce likelihood of operator reading error, they conceal the fact that precision of absorbance measurement is more difficult to obtain at low transmittances. It is apparent on a meter scale, linear for percent transmission, that the range of 1.0 to 2.0 absorbance occupies only 9 percent of the entire transmission scale; hence a technician may be leery of making readings in this range. But if a digitial display shows 1.931 for one absorbance reading and 1.968 for another, the operator is likely to interpret those readings as exact when, in fact, they are very probably quite inaccurate.

If an analog (continuously variable) signal is to be displayed digitally, an analog-to-digital conversion must occur. The accuracy with which the number displayed represents the original analog signal depends on the sophisitication of conversion circuitry; that accuracy is not absolute and can be quite poor.

As an example, we calculated the absorbance resolution of General Medical Sciences-Atomic Energy Commission (GeMSAEC) spectrophotometric systems. These systems employ analog-to-digital conversion circuitry with resolutions of $1 / 2^{10} \approx 0.1$ percent (ten bit) or $1 / 2^{12} \approx 1$ in 0.025 percent ( 12 bit). The best absorbance resolution deliverable by these two converters at several absorbance levels is shown in table 2. The difference in resolution between the two converters is apparent.

TABLE 2. Absorbance resolution for 10 and 12 bit analog-to-digital converters

\begin{tabular}{rrr} 
& \multicolumn{2}{c}{$\Delta A$} \\
\cline { 2 - 3 }$A$ & \multicolumn{1}{c}{$10 \mathrm{Bit}$} & \multicolumn{1}{c}{$12 \mathrm{Bit}$} \\
& & \\
0.0 & 0.00054 & 0.00014 \\
.3 & .00108 & .00027 \\
.7 & .00174 & .00043 \\
1.0 & .00546 & .00135 \\
1.5 & .01773 & .00436 \\
2.0 & .05798 & .01378
\end{tabular}

\section{E. Routine Instrument Performance Verification}

Although the necessity for initial verification of instrument performance is undeniable, relatively little has been done to incorporate separate instrument checks into the clinical laboratory quality control program. For example, this omission may permit an undetected malfunction to reduce the linear range of the spectrophotometer. Samples of normal concentration could still give accurate readings, but abnormally high concentration samples would produce readings falling outside the range of the instrument's linear response. To be sure, use of control samples simulating abnormal assay values would reveal this fault, but at the expense of complete re-run of all samples assayed preceding discovery of the difficulty.

Several wavelength and absorbance verification devices are available in readily usable form. Examples are the convenient Gilford (Gilford Instrument Laboratories, Oberlin, Ohio) calibration device for their 300-N Microsample Spectrophotometer, and the Arthur H. Thomas (Arthur H. Thomas Company, Philadelphia, $\mathrm{Pa}$.) didymium and holmium oxide filter glasses supplied with an adapter to fit into any sample carrier designed to hold standard $1 \mathrm{~cm}$ square cuvettes. However, it must be emphasized that it is appropriate use, and not mere possession of verification devices such as these, which standardize laboratory performance. 


\section{F. Operator Considerations}

Even an instrument system which is capable of meeting all necessary specifications will not reliably perform accurate analyses if that system is not designed to be easily operated in the proper manner. However, it is difficult to visualize a more ergonomically efficient spectrophotometer than the Gilford $300-N$. We have seen this instrument used to measure absorbances greater than 3.0; used to measure absorbance at two wavelengths without readjusting the zero setting; calibrated with the zero-set control and even operated from a cold start with no adjustment checks; all by experienced and presumably welltrained technicians who should know better. An undetermined but undoubtedly large contribution to poor quality control arises from improper operation of laboratory equipment, due to poor instrument design and poor operator understanding.

\section{Chemical Standardization}

Radin, in 1967, [22], reviewed the definition and use of standards and the standard materials available to the clinical chemist, and discussed the limitations of various so-called standards. His view that "there is actually very little in the way of official definitions of standards for the clinical chemist" was reinforced by a joint report of the National Academy of Sciences, National Research Council Subcommittee on Reference Materials [23]. The situation has improved since that time, as evidenced by the reports of Young and Mears [24], Meinke [25], and Zief and Michelotti [26]. The improvement has been due to the introduction by the National Bureau of Standards of Standard Reference Materials (SRM) for use in clinical chemistry. To July, 1971, these include cholesterol, urea, uric acid, creatinine, calcium carbonate, bilirubin, D-glucose, potassium chloride, tris (hydroxymethyl) aminomethane, tris (hydroxymethyl) aminomethane hydrochloride and glass filters for spectrophotometry.

The importance of the SRM's must not be underestimated; however, they represent a solution to only part of the problem. The clinical laboratory worker must be aware of errors which may occur despite the availability of SRM's; stability of standard solutions is particularly important. The variable stability of solutions of uric acid is well known, while Gowenlock [7] showed that in part, the lack of interlaboratory agreement for the determination of serum urea could be attributed to deterioration of aqueous urea standards containing no preservative. In a few cases, SRM's when used in aqueous solution are not ideal standards in clinical chemistry. Recently, we were investigating the determination of uric acid by an adaptation of the supposedly specific uricase neocuproine method [27], both manually and on the Beckman DSA-560. Unable to get $\mathbf{1 0 0}$ percent recovery of uric acid added to pooled human serum, we discovered that the slope of the standard uric acid curve (using SRM) can be varied according to the amount of bovine albumin added. When the concentration of bovine albumin is approxi- mately $7.0 \mathrm{~g} / 100 \mathrm{ml}, 100$ percent recovery of added uric acid is attainable.

A large number of routine clinical chemistry laboratories are using multichannel analyzers of both continuous flow and discrete sample type. Aqueous standard solutions containing a mixture of known amounts of all the substances commonly being determined in the routine clinical chemistry laboratory are not available. Multichannel machines are therefore being calibrated with commercially supplied calibration sera for which values for each determination have been assigned by the manufacturers. That wide differences may occur between the values obtained on the same machine with sera from different manufacturers, and even between lots from the same manufacturer, has been reported by Childress [28] and Helman, Reingold and Gleason [29]. These differences may occur for a variety of reasons, e.g., variation in weight or homogeneity of material. The Scientific and Technical Committee of the Association of Clinical Biochemists (A.C.B.) [30] reported variation in dry content from vials (all of the same lot number) of some manufacturer's control sera to be as high as 13.80 percent C.V. Six of the 10 brands investigated had coefficients of variation of over 2.0 percent. The deviation of the weight of the contents of these vials could be correlated to the deviation in analytical results obtained when these vials were reconstituted.

Data from our laboratory (table 3 ) shows the percentage deviation from the mean dry weight content of 10 vials of control sera compared with the percentage deviation from the mean analytical value for several determinations performed on the contents of each vial. The 10 vials were selected at random from a box of 40 vials (all of the same lot number); great care was taken to assure the correct amount of water was added to each vial. Replicate aliquots from each vial were analyzed in the same run. Excellent agreement was obtained between replicate analyses from the same vial for all the determinations. The variation in dry weight content of the vials is unacceptable, however. If the contents of each vial is representative of a homogeneous batch, one would expect the deviations from the mean assay values to be the same as the deviations of the weight values; this is not the case. Most batches of control sera from manufacturers are not subject to the large errors shown here. The large errors, however, pose the question of how frequently smaller and less easily recognized errors occur. The results showing variability of vial content were obtained on what was described by the manufacturer as control serum, not calibration serum. However, the same manufacturing standards are required for both control and calibration purposes.

Amador, Massod, and Franey [31] compared the values they obtained for aspartate aminotransferase in three commercial control sera with the values assigned by the manufacturer and found differences of 11 to 17 percent (possibly due to the manufacturer failing to provide sufficient details of the method they used to assign the values). In a more recent paper, Dobrow and Amador [32] reported a similar study for lactate 
TABle 3. Percentage deviation from the mean dry weight content $\bar{x}$ of 10 vials of control sera compared with the percentage deviation from the mean analytical value for several determinations performed on the contents of each vial

Percent deviation from $\bar{x}$ analytical value

\begin{tabular}{|c|c|c|c|c|c|c|}
\hline Vial & $\begin{array}{l}\text { Percent deviation } \\
\text { from } \bar{x} \text { dry } \\
\text { content }\end{array}$ & $\begin{array}{c}\text { Total } \\
\text { protein }\end{array}$ & Phosphate & Uric acid & Calcium & Magnesium \\
\hline 1 & -1.4 & -3.2 & -3.3 & -0.9 & -1.5 & 10.2 \\
\hline 2 & 1.9 & 1.4 & 2.7 & 5.6 & 0.7 & -5.5 \\
\hline 3 & 2.1 & 1.4 & 2.7 & 5.6 & 0.7 & -10.8 \\
\hline 4 & -2.1 & -1.7 & -3.3 & -3.0 & 0.7 & 10.2 \\
\hline 5 & -2.1 & -1.7 & -3.3 & -5.2 & -1.5 & 5.0 \\
\hline 6 & 1.8 & 4.4 & 5.7 & 3.4 & 0.7 & -5.5 \\
\hline 7 & -2.2 & -1.7 & -3.3 & -5.2 & -1.5 & 5.0 \\
\hline 8 & -2.2 & -1.7 & -3.3 & -5.2 & 0.7 & 5.0 \\
\hline 9 & 1.8 & 2.9 & 2.7 & 3.4 & 0.7 & -5.5 \\
\hline 10 & 2.3 & -1.7 & 2.7 & 3.4 & -1.5 & -5.5 \\
\hline
\end{tabular}

dehydrogenase, aspartate aminotransferase, alkaline and acid phosphatase, and amylase. They concluded that the activities stated by six of the eight manufacturers were doubtful or unacceptable in more than 50 percent of the sera tested. Bowers, Kelley and McComb [33] have shown that in some assay procedures, animal alkaline phosphatases (often used in commercial control sera) do not behave identically to human serum alkaline phosphatase.

These findings clearly indicate that currently available calibration sera should not be used directly as calibration materials for any analytical procedure.

Cali [34] suggested recently that SRM's make verification of commercial calibration sera possible; this statement is correct only if referee methods of analysis are available for use in conjunction with the SRM's. NBS will publish the first referee method for the determination of calcium shortly. The availability of referee methods and SRM's will do much to improve standardization in large laboratories. However, in small laboratories, where the problem is greatest, shortage of manpower and equipment does not permit analysis of calibration sera by referee methods. We suggest that NBS consider the production of reference sera with values for the common routine determinations assigned through use of their own SRM's and referee methods. These NBS reference sera would be used by laboratories to check commercial sera used for daily calibration of multichannel analyzers.

An alternative to the production of reference sera by NBS may be a certification program for commercial products. An example of the way in which an independent laboratory can be used for standard and reagent certification has been demonstrated by the cyanmethemoglobin certification program of the College of American Pathologists, as described by King and Willis [35].

In the meantime, the situation could be improved if the manufacturers used greater care in the preparation of calibration sera. When assigning values to calibration sera, they should indicate the exact methods used for this purpose. Values for aspartate aminotransferase and alkaline phosphatase, for example, are frequently said to have been assigned using the Karmen [36] or Bessey, Lowry, and Brock [37] methods, respectively. Almost certainly, modifications of these methods (with regard to substrate concentration buffer type, etc.) have been used, resulting in quite different activities from the original methods.

\section{Conclusions}

1. Spectrophotometric analyses in the routine clinical chemistry laboratory are performed within a unique framework.

2. The accuracy of results in clinical chemistry laboratories falls far below the level that should be expected.

3. Choice of instrumentation must be based on a thorough understanding of both the characteristics of the instrument and the assays which must be performed.

4. Except in the case of inadequate absorbance resolution, instrument availability does not generally limit clinical laboratory performance.

5. Materials for verification of instrument performance are often not available in a form suitable for routine use in the clinical laboratory.

6. Referee analytical methods are needed for use in conjunction with NBS standard reference materials.

7. The only materials currently available for calibration of multichannel analyzers are commercially prepared sera. Because of ill-defined methods of assigning values and poorly understood properties of enzymes from non-human sources, unverified use of these materials for calibration is highly undesirable.

8. The situation with regard to calibration sera could be improved by:

a. Greater care in the manufacture of calibration sera and provision of information concerning the exact method used to assign values to the material.

b. Certification of commercial calibration sera by an independent laboratory using NBS standard reference materials and referee methods.

c. Preparation by NBS of reference sera, to which values are assigned using standard reference materials 
and referee methods. Such reference sera could be used by laboratories to assign values to new batches of commercial sera.

9. The most sophisticated and versatile spectrophotometric system can yield standard results only when operated within its limitations. Chemical and serum reference materials must be used with due regard for their limitations. It is axiomatic then that, to understand and fully control what he can do, the laboratory operator must also understand what he cannot do with analytical apparatus and methods. He must be able to recognize bad results as bad and be able to interpret these mishaps to correctly evaluate laboratory performance just as the physician should interpret good laboratory results in diagnosis of the patient's physiologic performance.

\section{References}

[1] Belk, W. P., and Sunderman, F. W., A Survey of the Accuracy of Chemical Analyses in Clinical Laboratories, American Journal of Clinical Pathology 17, 853 (1947).

[2] Hendry, P. I. A., Proficiency Survey in Clinical Chemistry, 1961. Report of Adelaide Meeting, p. 8. College of Pathologists of Australia, 1961.

[3] Wootton, I. D. P., and King, E. J., Normal values for blood constituents: Inter-hospital differences, Lancet I, 470 (1953).

[4] Tonks, D. B., A Study of the accuracy and precision of clinical chemistry determinations in 170 Canadian laboratories, Clinical Chemistry 9, 217 (1963).

[5] Desmond, F. B., A clinical chemistry proficiency survey. New Zealand Medical Journal, 63, 716 (1964).

[6] Mitchell, F. L., Quality Control in a Large Laboratory, Proceedings of the Association of Clinical Biochemists, 4, 38 (1966).

[7] Gowenlock, A. H., Results of an interlaboratory trial in Britain, Annals of Clinical Biochemistry, 6, 126 (1969).

[8] Rand, Royden H., Practical spectrophotometric standards, Clinical Chemistry 15, 839 (1969).

[9] Edisbury, J. R., Practical Hints on Absorption Spectrometry (Hilger and Watts, London, 1966).

[10] Reule, Alfred, Testing spectrophotometric linearity, Applied Optics 7, 1023 (1968).

[11] Haupt, Geraldine W., An alkaline solution of potassium chromate as a transmittancy standard in the ultraviolet, Journal of the Optical Society of America 42, 441 (1952).

[12] Poulson, Richard E., Test methods in spectrophotometry: Stray-light determination, Applied Optics 3,99 (1964).

[13] Horecker, B. L., and Kornberg, Arthur, The extinction coefficients of the reduced band of pyridine nucleotides, Journal of Biological Chemistry 1 75, 385 (1948).

[14] Slavin, Walter, Photometric standard for ultraviolet-visible spectrophotometers, Journal of the Optical Society of America 52, 1399 (1962).

[15] Copeland, B. E., King, J., and Willis, C., The National Bureau of Standards carbon yellow filter as a monitor for spectrophotometric performance, The Journal of Clinical Pathology 49, 459 (1968).

[16] Colorimeters with Flowthrough Cells-A Critical Assessment of Four Instruments, Scientific Report No. 1. Association of Clinical Biochemists, England (1965).
[17] Colorimeters-A Critical Assessment of Five Commercial Instruments, Scientific Report No. 2. Association of Clinical Biochemists, England (1966).

[18] Ayres, Gilbert H., Evaluation of accuracy in photometric analysis. Analytical Chemistry 21, 652 (1949).

[19] Ringbom, Anders, Uber die Genauigkeit der Colorimetrischen Analysenmethoden I. Zeitschrift für Analytische Chemie 115,332 (1939).

[20] Willard, H. H., Merritt, L. L., and Dean, J. A., Instrumental Methods of Analysis (Van Nostrand, New Jersey, 1965).

[21] Amador, E., and Wacker, W. E. C., Serum glutamic-oxalacetic transaminase activity. A new modification and an analytical assessment of current assay techniques, Clinical Chemistry 8, 343 (1962).

[22] Radin, N., What is a standard, Clinical Chemistry 13, 55 (1967).

[23] Report by the Sub-Committee on Reference Materials, Committee on Analytical Chemistry Division of Chemistry and Chemical Technology, National Academy of SciencesNational Research Council: Standard reference materials as viewed by the laboratory supervisor-A status report, Analytical Chemistry 40,24 A (March 1968).

[24] Young, D. S., and Mears, T. W., Measurement and standard reference materials in clinical chemistry, Clinical Chemistry 14, 929 (1968).

[25] Meinke, W. W., Standard reference materials for clinical measurements, Analytical Chemistry 43, 28 A (May 1971).

[26] Zief, M., and Michelotti, F. W., Clinical chemistry: A challenge for high purity standards and reagents, Clinical Chemistry 17, 833 (1971).

[27] Morgenstern, S., Flor, R. V., Kaufman, J. H., and Klein, B., The automated determination of serum uric acid, Clinical Chemistry 12, 748 (1966).

[28] Childress, C. C.. An Improved Technique for the Standardization of the SMA 12 AutoAnalyzer, Advances in Automated Analysis, 1970, p. 167, Technicon International Congress.

[29] Helman, E. Z., Reingold, I. M., Gleason, I. O., Plea for standardization of commercial calibration materials for automated instruments, Clinical Chemistry 17, 1144 (1971).

[30] A note from Scientific and Technical Committee, Association of Clinical Biochemists, News Sheet, United Kingdom, p. 11 (Aug. 1971).

[31] Amador, E., Massod, M. F., Franey, R. J., Reliability of glutamicoxalacetic transaminase methods, American Journal of Clinical Pathology 47, 419 (1967).

[32] Dobrow, D. A., arid Amador, E., The accuracy of commercial enzyme reference sera, American Journal of Clinical Pathology 53. 60 (1970).

[33] Bowers, G. N., Kelley, M. L., and McComb, R. B., Precision estimates in clinical chemistry. Variability of analytic results in a survey reference sample related to the use of a nonhuman serum alkaline phosphatase, Clinical Chemistry 14, 595 (1967)

[34] Cali, P. J., SRM's and verification of commercial standards, Clinical Chemistry 18, 172 (1972).

[35] King, J. W., and Willis, C. E., Cyanmethemoglobin Certification Program of the College of American Pathologists, American Journal of Clinical Pathology 54, 496 (1970).

[36] Karmen, A., A note on the spectrophotometric assay of glutamicoxalacetic transaminase in human blood serum, Journal of Clinical Investigation 34, 131 (1955).

[37] Bessey, O. A., Lowry, O. H., and Brock, M. J., A method for the rapid determination of alkaline phosphatase with five cubic millimeters of serum, Journal of Biological Chemistry 164, 321 (1946). 